\title{
Prognostic impact of TID in regadenoson MPI: Some patients and certain events
}

\author{
Adrián I. Löffler, $M D{ }^{a}{ }^{a}$ and Jamieson $M$. Bourque, $M D, M H S^{a, b}$ \\ ${ }^{a}$ Cardiovascular Division and the Cardiac Imaging Center, Department of Medicine, University of \\ Virginia Health System, Charlottesville, VA \\ ${ }^{b}$ Department of Radiology, University of Virginia Health System, Charlottesville, VA
}

Received Sep 22, 2015; accepted Sep 23, 2015

doi: 10.1007/s12350-015-0299-9

\section{See related article, pp. 1147-1155}

Single-photon emission-computed tomographic (SPECT) myocardial perfusion imaging (MPI) has incremental diagnostic and prognostic value over exercise stress electrocardiography, and the assessment of transient ischemic dilation (TID) is a key contributor to its ability to detect higher risk patients. The diagnostic and prognostic role of TID has been evaluated extensively in those undergoing exercise MPI. ${ }^{1,2}$ However, approximately $50 \%$ of the $\sim 10$ million MPI studies performed annually in the US undergo pharmacologic MPI ${ }^{3}$ the majority receiving regadenoson for vasodilator stress due to its improved tolerability by patients, safety profile, and ease of administration. ${ }^{4}$ Given the high prevalence of this testing, the significance of TID in this population is of high importance. The diagnostic role of TID in regadenoson MPI has been assessed previously, but the prognostic impact has not determined.

In this issue of the Journal, Lester et al provide the first analysis of the prognostic utility of TID with regadenoson MPI. In this well-performed study, Lester et al assessed the prognostic impact of an abnormal TID ratio of 1.33 on a composite of cardiac death, myocardial infarction (MI), or late coronary revascularization (CR) in 887 patients over a mean of 29 months; $75 \%$ of the cohort had an abnormal perfusion pattern. TID was

\footnotetext{
Reprint requests: Adrián I. Löffler, MD, Cardiovascular Division and the Cardiac Imaging Center, Department of Medicine, University of Virginia Health System, Box 800158, 1215 Lee Street, Charlottesville, VA 22908; al2ys@hscmail.mcc.virginia.edu

J Nucl Cardiol 2016;23:1156-9.

$1071-3581 / \$ 34.00$

Copyright (c) 2015 American Society of Nuclear Cardiology.
}

found in $6 \%$ of this population. After multivariable adjustment, TID had a hazard ratio of 1.92 for predicting the composite endpoint. Although the predominant conclusion of the study is that TID has prognostic importance, there are two significant additional considerations. The first is that TID was only prognostic in this cohort in those with abnormal MPI. In those with normal perfusion, there was no association. The second is that the prognostic significance was driven by late CR, rather than the hard cardiac events of cardiac death and nonfatal MI. Given these findings, we will discuss the potential mechanism for TID in vasodilator MPI and its potential effect on specific cardiac events, the diagnostic and prognostic significance of TID with respect to concurrent MPI abnormalities, and future directions for research and clinical application of TID in vasodilator MPI.

\section{TID: DEFINITION AND POTENTIAL MECHANISM IN VASODILATOR MPI}

TID refers to an enlarged left ventricular (LV) cavity measured in post-stress scintigraphy images relative to resting images. Lester et al appropriately highlight the importance of selecting an appropriate TID cut-off ratio. The cut-off ratio used to define TID has varied in the literature based on stress modality, nuclear tracer, imaging protocol, and other technical factors. ${ }^{5}$ The authors derived a cut-off ratio of 1.33 in this study, as this value encompassed $95 \%$ of the cohort population.

TID has been shown to independently provide diagnostic utility in predicting severe and extensive coronary artery disease (CAD) and overall poor prognosis. However, there is a controversy regarding its appropriate application across varying clinical settings. Some of these inconsistent findings may be explained by the underlying mechanism of TID, which is still debated. One hypothesized pathophysiologic mechanism is diffuse subendocardial hypoperfusion resulting in 
reduced radiotracer uptake in the subendocardial zone, thus, making the appearance of an enlarged LV cavity. ${ }^{5}$ An alternative theory is ischemia resulting in reduced LV function with resultant elevated end-systolic volume. The true mechanism may combine these two theories. ${ }^{6,7}$ These potential mechanisms can explain why TID is not always associated with severe CAD on angiography or poor prognosis. Patients who have other causes of diffuse subendocardial ischemia, such as hypertensive heart disease and LV hypertrophy or hypertrophic cardiomyopathy, can have TID without obstructive CAD. Other potential mechanisms for falsely increased TID ratios include suboptimal technique, errors in slice selection for tomographic analysis, patients with small LV ventricles, different doses of administered radionuclide, or motion artifacts. ${ }^{5}$ Thus, it is important to appropriately identify these factors prior to assessing for TID. Regadenoson is a selective adenosine A2A receptor agonist which causes sinus tachycardia. It has been proposed that the mechanism for tachycardia is mainly due to sympathoexcitation rather than being baroreflex mediated ${ }^{8,9}$ as the heart rate response is blunted among diabetics. ${ }^{9}$ Thus, one would expect this to generate diffuse subendocardial hypoperfusion in certain clinical scenarios, which is one of the proposed underlying mechanisms of TID.

\section{TID AND ITS SIGNIFICANCE ON CORONARY ANGIOGRAPHY}

Several studies have shown that TID on SPECT MPI is specific but not sensitive for significant multivessel or left main disease on coronary angiography. Weiss et al examined a cohort undergoing exercise thallium-201 SPECT MPI and noted high specificity (95\%) but lower sensitivity $(60 \%)$ for identifying patients with multi-vessel critical stenosis $(\geq 90 \%)$ using a TID threshold of $>1.12$. They found TID to be more specific than multiple perfusion defects. ${ }^{1}$ Mazzanti et al studied 54 patients with MPI abnormalities on exercise dual-isotope TC-99m/TI-201 SPECT and showed that TID in this setting of abnormal MPI was associated with $71 \%$ sensitivity and $95 \%$ specificity for severe and extensive CAD defined as a $\geq 90 \%$ stenosis of the proximal LAD or $\geq 2$ vessel disease. ${ }^{2}$ Similar diagnostic information has been seen in patients with vasodilator MPI. Chouraqui et al performed a small retrospective cohort study that found high specificity for TID in the identification of $\geq 2$ vessel $(87 \%)$ or 3 -vessel $(85 \%)$ CAD using a threshold of $\geq 1.12$ in 73 patients undergoing dipyridamole stress-redistribution thallium-201 scintigraphy and coronary angiography. They did not specifically analyze the subgroup with perfusion abnormalities. ${ }^{10}$ Takeishi et al found an association of left main or three-vessel coronary disease with a LV dilation ratio of $1.13 \pm 0.04$ in 75 patients who underwent adenosine TI-201 single-photon emission tomography with CAD defined by coronary angiography. ${ }^{11}$ Both of these studies were too small to specifically assess the population with perfusion abnormalities.

Contemporary studies in patients with abnormal MPI have found a similar diagnostic utility of TID. Xu et al performed a contemporary analysis of TID in 547 patients undergoing SPECT MPI (215 with correlating coronary angiography). They found an increased sensitivity for severe CAD ( $\geq 70 \%$ stenosis in proximal left anterior descending (pLAD) artery or left main (LM), or $\geq 90 \%$ in $\geq 2$ vessels), in those with TID (cut-off 1.19) and perfusion abnormalities. ${ }^{12}$ Petretta et al studied a cohort of diabetic patients with available coronary angiography data underdoing SPECT MPI. In patients with a summed stress score (SSS) between 3 and 7, TID increased the sensitivity for diagnosing severe CAD from $71 \%$ to $77 \% .^{13}$

Recent contemporary studies on TID in otherwise normal MPI have not found TID to independently identify severe and extensive $\mathrm{CAD}$ as recently reviewed. ${ }^{14}$ Valdiviezo et al studied a cohort of normal perfusion imaging and found no association of TID and multi-vessel disease. ${ }^{15}$ Mandour et al found that TID had predictability in patients with perfusion abnormalities but not in the setting of normal perfusion. ${ }^{16}$

To date only two studies have assessed the diagnostic utility of TID in regadenoson SPECT MPI and have demonstrated conflicting findings. Katz et al found that patients with an abnormal TID during regadenoson gated SPECT MPI had an adjusted odds ratio of 3.4 for multi-vessel disease. ${ }^{17}$ Golzar et al recently studied the ability of TID in regadenoson-stress MPI to identify severe CAD and failed to do so even when adding clinical and perfusion data. The authors felt that perhaps the decreasing prevalence of CAD could explain this negative finding. ${ }^{18}$

\section{EFFECT OF TID ON PROGNOSIS}

While many historical studies have shown the predictive utility of TID for identifying severe CAD, fewer studies have focused on its ability to predict cardiac events. Lester et al demonstrated that TID with regadenoson MPI independently predicts a composite of cardiac events (cardiac death, non-fatal MI, and late $\mathrm{CR}$ ); however, the association was largely driven by late $\mathrm{CR}$. When assessing cardiac death and non-fatal MI separately, there was no association with the presence or absence of TID. A review of the literature reveals that the effect of TID on prognosis depends on the presence of perfusion defects and associated comorbidities and demographics. 
McClellan et al studied the prognostic implication of TID on dipyridamole technetium-99 SPECT imaging at a mean follow-up of 12.8 months. Those with TID had an $11.4 \%$ occurrence of cardiac death or non-fatal MI vs $1.9 \%$ in patients with normal cavity size. The group with the highest risk had both large perfusion defects and cavity dilation. ${ }^{19}$ Rischpler et al found a lower overall survival probability with TID in a cohort with suspected or known CAD who underwent restdipyridamole 82 -Rb PET. ${ }^{20}$ Petretta et al showed that adding TID to other clinical and stress testing variables improves the ability to predict cardiac death and nonfatal MI in patients with diabetes mellitus. ${ }^{21}$ Interestingly, in a predominately female Middle Eastern cohort undergoing SPECT MPI, summed difference score and abnormal EF were associated with cardiac events but TID showed no relationship (although there was a trend toward increased cardiac events). ${ }^{22}$

Abidov et al studied the prognostic significance of TID in normal MPI and found an association with adverse events driven mainly by revascularization. ${ }^{23}$ When studying subjects with normal perfusion on MPI predominately with exercise stress, Doukky et al demonstrated that TID was associated with cardiac death or MI in high-risk subjects (those with known CAD or diabetes) but not those without CAD or diabetes. ${ }^{24}$

In this issue of the Journal, Lester et al used a cohort enriched with high-risk scans to look at prognosis of TID with regadenoson MPI. Although constructed artificially, the population assembled by Lester et al helps assess if declining prevalence is the reason for the lack of clinical utility in TID shown by Golzar et al. ${ }^{18}$ In contrast to prior studies showing an association between TID and hard cardiac events, Lester et al found no prognostic benefit in these outcomes. It is possible that this finding is due to the large number of patients who underwent early revascularization (within 90 days of MPI) in those with TID (22\% vs $11 \%, P=0.04)$ potentially protecting them from cardiac death or non-fatal MI events.

Of note, Kaplan-Meier analysis in Fig. 2, the analysis by Lester et al showed a non-significant trend toward an improved survival free of the composite endpoint. Interestingly, Valdiviezo et al had an almost identical finding. Future research should focus on larger studies or a meta-analysis to see if a protective effect of TID is demonstrated in lower risk patients with normal perfusion. The underlying pathophysiologic mechanism for this finding is not clear.

\section{RESEARCH CHALLENGES AND FUTURE DIRECTIONS}

The analysis by Lester et al adds value to the current controversy for the clinical application for TID, highlighting that TID found on regadenoson SPECT adds prognostic value in certain clinical settings. In low risk patients with no high-risk comorbidities and no perfusion abnormalities on MPI, TID does not appear to worsen outcomes and may actually have a protective effect. Larger studies assessing TID in this population are needed. TID in patients with normal exercise MPI but higher risk comorbidities appears to be associated with poorer outcome as highlighted by Doukky et al. ${ }^{24}$ Studies reproducing these findings in pharmacologic stress MPI are needed. Studies utilizing other clinical variables such as calcium scoring to help determine which patients would most benefit from coronary angiography would be beneficial. ${ }^{14}$ Abnormal perfusion on MPI and TID appears to increase the diagnostic likelihood of severe and extensive CAD and poorer outcomes, although not all studies are concordant. ${ }^{18}$ Given that diffuse subendocardial ischemia may be a potential mechanism for TID, future studies should associate findings of concentric LV hypertrophy on echo data as a potential confounder. Future larger studies should also assess prognosis in cohorts typically presenting for regadenoson SPECT in patients with abnormal perfusion MPI and include subgroup analysis in those with and without CAD, diabetes, and CKD. It would be interesting to study the prognostic effect of TID and abnormal MPI in patients found to have no obstructive $\mathrm{CAD}$ on coronary angiography. If we are able to demonstrate in these studies that TID is only a poor prognostic indicator in patients with abnormal MPI, then perhaps utilizing stress-only imaging protocols in patients that can not exercise may be appropriate and financially optimal.

\section{Acknowledgments}

Dr. Bourque receives research grant support from Astellas Pharma.

\section{References}

1. Teddy Weiss A, Berman DS, Lew AS, Nielsen J, Potkin B, Swan $\mathrm{HJC}$, et al. Transient ischemic dilation of the left ventricle on stress thallium-201 scintigraphy: A marker of severe and extensive coronary artery disease. J Am Coll Cardiol 1987;9:752-9.

2. Mazzanti M, Germano G, Kiat H, Kavanagh PB, Alexanderson E, Friedman JD, et al. Identification of severe and extensive coronary artery disease by automatic measurement of transient ischemic dilation of the left ventricle in dual-isotope myocardial perfusion SPECT. J Am Coll Cardiol 1996;27:1612-20.

3. Zoghbi GJ, Iskandrian AE. Selective adenosine agonists and myocardial perfusion imaging. J Nucl Cardiol 2012;19:126-41.

4. Hage FG, Ghimire G, Lester D, McKay J, Bleich S, El-Hajj S, et al. The prognostic value of regadenoson myocardial perfusion imaging. J Nucl Cardiol. 2015. doi:10.1007/s12350-014-0050-y. 
5. McLaughlin MGDP. Transient ischemic dilation: A powerful diagnostic and prognostic finding of stress myocardial perfusion imaging. J Nucl Cardiol 2002;9:663-7.

6. Marcassa C, Galli M, Baroffio C, Campini R, Giannuzzi P. Transient left ventricular dilation at quantitative stress-rest sestamibi tomography: Clinical, electrocardiographic, and angiographic correlates. J Nucl Cardiol 1999;6:397-405.

7. Bestetti A, Di Leo C, Alessi A, Triulzi A, Tagliabue L, Tarolo GI. Post-stress end-systolic left ventricular dilation: A marker of endocardial post-ischemic stunning. Nucl Med Commun 2001; 22:685-93

8. Dhalla AK, Wong M, Wang W, Biaggioni I, Belardinelli L. Tachycardia caused by A2A adenosine receptor agonists is mediated by direct sympathoexcitation in awake rats. J Pharmacol Exp Ther 2006;316:695-702.

9. Hage FG, Heo J, Franks B, Belardinelli L, Blackburn B, Wang W, et al. Differences in heart rate response to adenosine and regadenoson in patients with and without diabetes mellitus. Am Heart J 2009;157:771-6

10. Chouraqui P, Rodrigues EA, Berman DS, Maddahi J. Significance of dipyridamole-induced transient dilation of the left ventricle during thallium-201 scintigraphy in suspected coronary artery disease. Am J Cardiol 1990;66:689-94.

11. Hozumi T, Yoshida K, Akasaka T, Asami Y, Ogata Y, Takagi T, et al. Noninvasive assessment of coronary flow velocity and coronary flow velocity reserve in the left anterior descending coronary artery by doppler echocardiography: Comparison with invasive technique. J Am Coll Cardiol 1998;32:1251-9.

12. Xu Y, Arsanjani R, Morgan C, Hyun M, Lemley M, Fish M, et al. Transient ischemic dilation for coronary artery disease in quantitative analysis of same-day sestamibi myocardial perfusion SPECT. J Nucl Cardiol 2012;19:465-73.

13. Petretta M, Acampa W, Daniele S, Petretta MP, Nappi C, et al. Transient ischemic dilation in SPECT myocardial perfusion imaging for prediction of severe coronary artery disease in diabetic patients. J Nucl Cardiol. 2012;20:45-52.

14. Jamieson M, Bourque MD. MHS. Contemporary relevance of TID: Based on the company it keeps. J Nucl Cardiol 2015;22:5358.

15. Valdiviezo C, Motivala AA, Hachamovitch R, Chamarthy HM, Navarro PC, et al. The significance of transient ischemic dilation in the setting of otherwise normal SPECT radionuclide myocardial perfusion images. J Nucl Cardiol 2011;18:220-9.
16. Mandour Ali MA, Bourque JM, Allam AH, Beller GA, Watson DD. The prevalence and predictive accuracy of quantitatively defined transient ischemic dilation of the left ventricle on otherwise normal SPECT myocardial perfusion imaging studies. J Nucl Cardiol 2011;18:1036-43.

17. Katz JS, Ruisi M, Giedd KN, Rachko M. Assessment of transient ischemic dilation (TID) ratio in gated SPECT myocardial perfusion imaging (MPI) using regadenoson, a new agent for pharmacologic stress testing. J Nucl Cardiol 2012;19:727-34.

18. Golzar Y, Olusanya A, Pe N, Dua SG, Golzar J, et al. The significance of automatically measured transient ischemic dilation in identifying severe and extensive coronary artery disease in regadenoson, single-isotope technetium- $99 \mathrm{~m}$ myocardial perfusion SPECT. J Nucl Cardiol 2015;22:526-34.

19. McClellan JR, Travin MI, Herman SD, Baron JI, Golub RJ, Gallagher JJ, et al. Prognostic importance of scintigraphic left ventricular cavity dilation during intravenous dipyridamole technetium-99m sestamibi myocardial tomographic imaging in predicting coronary events. Am J Cardiol 1997;79:600-5.

20. Rischpler C, Higuchi T, Fukushima K, Javadi MS, Merrill J, Nekolla SG, et al. Transient ischemic dilation ratio in 82Rb PET myocardial perfusion imaging: Normal values and significance as a diagnostic and prognostic marker. J Nucl Med 2012;53:723-30.

21. Petretta M, Acampa W, Daniele S, Petretta MP, Plaitano M, Cuocolo A. Transient ischemic dilation in patients with diabetes mellitus: Prognostic value and effect on clinical outcome after coronary revascularization. Circulation 2013;6:908-15.

22. Chavoshi M, Fard-Esfahani A, Fallahi B, Emami-Ardekani A, Beiki D, Hassanzadeh-Rad A, et al. Assessment of prognostic value of semiquantitative parameters on gated single photon emission computed tomography myocardial perfusion scintigraphy in a large middle eastern population. Indian $\mathrm{J}$ Nucl Med 2015;30:233-8

23. Abidov A, Bax JJ, Hayes SW, Hachamovitch R, Cohen I, Gerlach $\mathrm{J}$, et al. Transient ischemic dilation ratio of the left ventricle is a significant predictor of future cardiac events in patients with otherwise normal myocardial perfusion SPECT. J Am Coll Cardiol 2003;42:1818-25.

24. Doukky R, Frogge N, Bayissa YA, Balakrishnan G, Skelton JM, Confer $\mathrm{K}$, et al. The prognostic value of transient ischemic dilatation with otherwise normal SPECT myocardial perfusion imaging: A cautionary note in patients with diabetes and coronary artery disease. J Nucl Cardiol 2013;20:774-84. 